\title{
Recent developments in infrastructure and capacity building at the Teaching Hospital, Karapitiya (THK), Sri Lanka towards improving care given to patients with trauma and emergencies
}

\author{
PL Ariyananda ${ }^{1}$, K Jayasekera $^{2}$

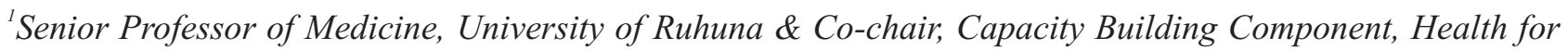 \\ South Project, Government of Victoria, \\ ${ }^{2}$ Physician-in-charge, ETU and Visiting Physician OPD, Teaching Hospital Karapitiya, Galle.
}

e-mail address of the corresponding author,Prof.PLAriyananda: ariyananda@sltnet.1k

\section{Introduction}

Sri Lanka was devastated by the Asian Tsunami of 2004 December which took lives of nearly 40,000 people. Galle District too had a substantial share of the losses amounting to over 5,000 lives. Although most of these deaths were not preventable, a significant number of deaths could have been prevented if THK was in a state of disaster preparations.

In the wake of the tsunami disaster among many donations that poured into Sri Lanka, an aid package of around 500 million Sri Lankan rupees was donated by the Government of Victoria to construct an Emergency and Trauma Centre (ETC) for THK under a project named 'Health for South'. The Government of Sri Lanka signed a Memorandum of Understanding with the Government of Victoria in February 2005 to enable implementation of several aid packages including the 'Health for South' project.

\section{Shortcomings in facilities necessary for emergency and trauma care at THK}

A feasibility study was carried out at the THK in 2006 by the Government of Victoria before drawing up a proposal on the aid package. The study disclosed several deficiencies in facilities available to cater medical and surgical emergencies as outlined below ${ }^{1,2}$.

- The hospital lacked basic facilities for effective triage and reception of accident and emergency patients and there were inevitable delays in assessment and diagnosis of such patients. There were only two trolleys available for urgent care and resuscitation in the Emergency treatment Unit (ETU) of the Out-patient Department (OPD) at the time of the tsunami.

- The hospital had a shortage of Intensive Care Unit (ICU) facilities and had only 8 adult ICU beds. Many patients who would benefit from ICU care were unable to access this facility.

- Operation Theatres and theatre facilities too were inadequate. This was a serious drawback in the management of complex orthopaedic problems such as compound fractures or fractures requiring internal fixation. Long unacceptable delays of several days occurred before definitive treatment risking complications such as bone infections. Further, it was noted that the existing internallycirculating air conditioning system paused a higher risk of infection during orthopaedic operations in contrast to the desirable laminar flow type of air conditioning.

- Lack of a dedicated area for management of spinal and neurotrauma was a notable shortcoming.

- Lack of dedicated facilities for resuscitation and assessment of acute paediatric emergencies.

- Lack of dedicated facilities for assessment of psychiatric emergencies.

The current model of care in THK and opportunities for development

The existing model of care for trauma and 
emergencies at THK is similar to what is available in other major hospital in Sri Lanka except the Colombo National Hospital. This is a model of care where hardly any assessment or treatment is done in the outpatient department. The patient arrives in the casualty medical or surgical ward after some delay before assessment and treatment of emergency care. Such delays can contribute to increase in mortality and morbidity. Furthermore, according to this model of care the patient is initially seen by an intern house officer with little experience whereas it is desirable for such undifferentiated acutely ill patients to be seen by a doctor with more experience.

In Emergency Treatment Centers that function in developed countries, patients with trauma or emergencies are seen by doctors who are specialized in Emergency Medicine on arrival. Such a model helps to reduce the mortality and morbidity of patients. In Sri Lanka, currently there are no specialists in Emergency Medicine. However, the Postgraduate Institute of Medicine, Colombo (PGIM) is strongly considering developing Emergency Medicine as a specialty in the near future.

\section{Health for South Project}

The Victorian aid package for development of an Emergency Trauma Centre at THK endorsed the following recommendations and the infrastructure would be expected to fall in line with models operating in developed countries. What is envisaged in the ETC Project can be considered in two major areas: Infrastructure Development and Capacity Building ${ }^{3}$.

Infrastructure Development component can be considered under four areas: Accidents and Emergency section, Urgent Theatre Care Complex, ICU and Short-stay Wards. Most of the equipment and devices necessary for the ETC will be provided by the Government of Victoria with the short fall being supplemented by the Ministry of Health.

\section{- Accidents and Emergency:}

This will include a triaging area, emergency resuscitation bays, separate assessment areas for adults and children, mini-theatre, laboratory, Xray facility, CT scanning facility and ambulance bays.

\section{- Urgent Theatre Care Complex}

This will include four theatres - two general surgical, one each of neurosurgical and orthopaedic

\section{- Intensive Care Unit}

This will service eight beds.

\section{- Short-stayWards}

There will be two wards with a total of 56 shortstay beds for rapid patient turnover within 24 hours.

The Capacity Building Programme (CBP) was designed and executed by the staff of ETC of The Alfred Hospital, Melbourne, Victoria with a staff that had extensive experience in managing patients with emergencies and trauma care ${ }^{4,5}$. The ETC of The Alfred Hospital, Melbourne, Victoria is a centre of excellence and considered non-arguably the best ETC in the Southern Hemisphere. The CBP was conducted over period of 15 months from April 2007 to June 2008. Six training modules were conducted in the aspects of emergency care: trauma, the critically ill patient (non trauma), paediatric emergencies, medical emergencies, surgical emergencies and disaster preparedness. Emphasis was given to trauma reception and resuscitation in all the modules. Four of the six modules were conducted over a 3 week period and the other two modules were conducted over a period of one to two weeks. All participants were evaluated at the end of each module. The CBP was conducted by a visiting team from Victoria comprising one emergency physician and two critical care/trauma nurses.

The following THK staff was trained at the ETC of the Alfred Hospital in Melbourne. In December 2007 the Physician-in-charge of ETU and an ETU nurse and in June 2008 a surgeon and an anaesthetist underwent training for a period of 3 weeks. In June 2008 a nurse from the ETU underwent training for a period of 3 months.

The expected outcomes of Health for South Project include;

- Improvement in assessment, resuscitation and stabilization of critically ill patients presenting to THK.

Galle Medical Journal, Vol 14: No. 1, September 2009 
- Improvement of patient outcome by reducing delay in initiating treatment.

- Reducing unnecessary admissions and thereby reducing overcrowding of wards.

- Reducing duration of hospital stay.

- Improvement to patient admission process and decision making through training programmes.

- Increasing the operating theatre capacity and surgical facilities which will enable timely treatment, in particular orthopaedic cases.

- Developing the capacity of THK to handle disaster and mass casualities.

- Developing a nurse-based triaging system.

\section{Scope of ETC for expansion of its services in the future}

In addition to outcomes listed above the ETC should be a facility which

a) coordinates pre-hospital care

b) provide undergraduate and postgraduate training for doctors, nurses and paramedical personnel.

\section{Pre-hospital care:}

In Sri Lanka there are no trained paramedical personnel to go in the ambulance to provide triaging and resuscitation on-site for emergency calls. THK does not have a dedicated fleet of ambulances to provide pre-hospital care. These shortcomings have to be addressed if we are to improve pre-hospital services in order to bring down mortality and morbidity due to trauma and emergencies. Early retrieval and rescue gives a golden window of opportunity to save lives and every effort should be made to make use of this opportunity.

\section{Services as a training Centre:}

ETC will be equipped with a lecture theatre to enable formal in-house teaching. However, most of the training expected to be imparted to doctors and nurses would be by way of on-site hands-on training. Such training will be given by specialists who would work as leaders of a multidisciplinary team. The principles of triaging and protocol driven management is expected to be executed in the ETC. The establishment of the ETC will help to give focused training to trainees of the Diploma programme in Critical Care that was recently commenced by the PGIM. Emergency medicine is a new specialty with a novel approach where specialists who are specifically trained to handle undifferentiated patients as they arrive in the hospital thereby initiating the most appropriate treatment at the earliest opportunity ${ }^{6}$. PGIM hopes to commence a specialist programme in emergency medicine in few years time and the ETC is expected to play a key role as a training centre for this programme.

\section{Concluding remarks}

The construction of the ETC was started in April 2009 and the project is expected to be completed in mid-2010. The Ministry of Health is expected to provide some equipment, logistical support and additional doctors, nurses and other staff that is necessary to run the ETC smoothly and effectively. We hope to see specialists in a multidisciplinary team managing patients with multisystem trauma or multiple organ dysfunction in resuscitation bays of the ETC at THK by the year end of 2010. Such resuscitations are expected to be immediately followed by quality treatment within the ETC until an ICU bed is available for the patient in the Hospital. As such the ETC is expected to bring down mortality and morbidity in patients with emergencies and trauma significantly.

The trigger to set up of the ETC was the Asian Tsunami of 2004. Although tsunamis come once in every 500 years or so we need to realize that we have to save the lives of patients who come with other illness every day. We hope that the ETC of THK will be a centre of excellence and a good model for delivery of emergency care in Sri Lanka.

\section{References}

1. Fitzgerald M. The post tsunami development of emergency services at Karapitiya Hospital, Galle, Sri Lanka. Report to the Department of Premier and Cabinet, State Government of Victoria and World Vision, Australia, Melbourne. 2005. 
2. Miller C. Project report: Health for the South. Report on site visit.2005.

3. Williams S, Fitzgerald M, Ariyananda PL, O'Reilly G (Capacity Building Steering Committee). Health for the South - Emergency Trauma Centre Project - Capacity Building Component - Terms of Reference. Melbourne: Unpublished, 2006.

4. O'Reilly G, Fitzgerald M, Ariyananda PL et al. In the wake of Sri Lanka's tsunami: the health for the south capacitybuilding project. Emerg. Med. Australas 2008; 20: 175-9.
5. O'Reilly G, Fitzgerald M, Ariyananda PL et al. Trauma reception and resuscitation in Sri Lanka: Health for South Capacity-Building project. Emerg. Med. Australas 2009; 21: $147-152$.

6. Tan G, Gunasekera C, Curran S. Emergency Medicine in Sri Lanka: The inevitable solution to a new specialty. Emerg. Med. Australas. 2008; 20: 81-4. 